\title{
Research and Design of Context-Aware English Knowledge Push APP
}

\section{Based on LBS}

\author{
Changpin Chen ${ }^{1, \mathrm{a}}$, Siwen $\mathrm{Guo}^{2, \mathrm{~b}}$, Chuwen $\mathrm{Liu}^{3, \mathrm{c}}$ and Xiande Zhou ${ }^{4, \mathrm{~d}}$ \\ ${ }^{1}$ Labcenter,Guangzhou University, Guangzhou, Guangdong 510006, China \\ ${ }^{2}$ School of Computer Science and Educational Software, Guangzhou University, Guangzhou, \\ Guangdong 510006, China \\ ${ }^{3}$ School of Computer Science and Educational Software, Guangzhou University, Guangzhou, \\ Guangdong 510006, China \\ ${ }^{4}$ School of Computer Science and Educational Software, Guangzhou University, Guangzhou, \\ Guangdong 510006, China \\ achenchpkiko@gzhu.edu.cn, ${ }^{\mathrm{a}}$ guosiwen@tsinghua.org.cn, 'shuangyue17@126.com, \\ d212666292@qq.com
}

Keywords: LBS; POI; context-aware; OWL; knowledge push

Abstract. LBS make it possible that the computer system can be context-aware for learners and push the relevant knowledge of English to them. As long as the relationship between these situations and the information of the specific location is built, through the database and query of POI which is supported by LBS, the system can realize context-aware automatically. The system will build the English knowledge database for each context, which include English words, English phrase and English sentences. As soon as the system aware the context where the users are, the system will extract data from context-knowledge database automatically, and then filter the English Knowledge, which the users need in a smart way, according to the user's interests and the records of user's history access. Through the message push service, the system will promptly push the English knowledge to help the users to the informal study.

\section{Introduction}

The traditional way of learning English focused on classrooms and books is form-single, lack of context information, result in difficult to apply their knowledge. In fact, English as a communication tool to understand and master the use of English in different contexts is an effective way to achieve their knowledge.

The approach that use micro-mobile terminals for informal learning to help learning and research for person has been proved to be effective by more and more practitioners. ${ }^{[1]}$ The concept on knowledge push intended to provide the appropriate knowledge to ones based on whose knowledge needs, was dreamed up first by Rafaela, the Editor-in-Chief of "Information Week" in USA, when he introduced Microsoft's knowledge push system. ${ }^{[2]}$ The "smart phone plus knowledge push" mode is an effective means for informal learning.

At the same time, the LBS make it possible that the computer system can be context-aware for learners and push the relevant knowledge of English to them. Location-Based Service (LBS) acquires the location information of the mobile terminal user through the mobile carrier's radio communication network (such as GSM network, CDMA network) or external positioning mode 
(such as GPS). ${ }^{[3]}$ LBS are position-sensitive, which can be combined with context-awareness. We can extract automatically the information needed by user and suggest to one, according to the time and space environment information around one, in conjunction with one's personal interest preference, from the user's point of view. So we can serve users with the most relevant and appropriate ways. An example on LBS-based context-aware is that once a user enters a western-style restaurant, the system will push automatically English vocabulary and commonly used dialogue relevant with western-style restaurant.

\section{The Status Quo and Appropriateness}

The Present Situation of Android App on English Learning in China. Context refers to the social environment where person participate in activities. It is the specific conditions for people to make social behavior. ${ }^{[4]}$ Dey, the expert on context-aware research thinks a system with context-aware capacity can provide relevant information and services according to the context to users based on their perceived needs. ${ }^{[5]}$ According to this definition, I think that the context-aware knowledge push system based on LBS can detect automatically the user location using sensor device, determine the situation in which the user is, further infer the user's actual or potential learning needs, and filter and push the knowledge to user to promote informal learning, even though the user does not issue a service request.

In the mainstream android markets in China, English-learning related apps cover electronic dictionary, translation tools, etc. There is no English learning app based on LBS technology at present.

Appropriateness of Context-aware English Knowledge Push Based on LBS. According to CNNIC statistics, as of June 2016, China's mobile phone users have reached 656 million, and the population of Internet users who use the mobile phone is increase from $90.1 \%$ of the end of 2015 to 92.5\%. ${ }^{[6]}$ The smart phones support many kind of positioning technology, such as GPS, indoor wireless orientation, etc., which can be used to get the user's current location in time.

In reality, the activities can be classified according to the social activities, so as to define the different contexts, such as restaurants, supermarkets, airports, libraries, etc. The classification of these contexts can also be broken down, such as the restaurants can be divided into western restaurants, Chinese restaurants, coffee shops, etc. As long as the relationship between these situations and the information of the specific location is built, through the database and query of POI which is supported by LBS, the system can realize context-aware automatically. We need build context-related English knowledge database including words, phrase and sentences.

As soon as the system aware the context where the users are, the system will extract data from context-knowledge database automatically, and then filter the English Knowledge, which the users need in a smart way, according to the user's interests and the records of user's history access.

Through the message push service, the system will promptly push the English knowledge to help the users to the informal study. When the users receive the English knowledge, the users can feedback, such as whether they like, etc.

\section{Ontology Model of English Knowledge}

Building the Ontology Model. It is an effective way to cultivate people's communication ability if people can use English knowledge in the real communication situation which can be automatically cognized with the context awareness technology based on LBS. 
In order to improve the relevance and aptitude degree, so that the resources can be used to improve user's learning efficiency, the resources must be not only relative with user's context and hobbies, but also well-organized when system pushes resources which contain knowledge points to users.

OWL can be used to express the relationship between terms and terms in lexical system, and it is an ontological knowledge representation method. As far as the expression of meaning and semantics, OWL is more powerful than XML, RDF, and RDF-S, and other languages. It has the most powerful ability to express information on the Web. ${ }^{[7]}$ OWL provides three sub-languages: OWL Lite, OWLDL, and OWLFull, which are increasingly expressive. OWL Full can be used together with RDF-S. Same as RDF-S, it does not enforce a strict distinction between classes, attributes, individuals, and data values. ${ }^{[8]}$ In terms of grammar, OWL follows the XML syntax as well as the XML-S. ${ }^{[9]}$ It is simple, and easier to understand. The scalability of OWL is excellent, and can continuously improve the definition of the class, attribute or instance.

There have been a lot of development and maintenance tools for XML In the field of software engineering. There have been many edit tools for the OWL language, such as Jena, protege, etc., after OWL has been defined as the recommended standard.

This paper applies OWL to representations of English knowledge and complies with the syntax constraints of OWL Full. The knowledge of English used in the system is edited and maintained by protégé. The model is showed as Fig.1.

The process of knowledge representation based on OWL is: firstly, it is necessary to make the abstract form of knowledge by the object-oriented abstraction method. Secondly, build the type declaration document (DocType.owl) to define the knowledge representation syntax in domain. Finally, build OWL knowledge document (DocKnowledge.owl) to define the instances of these classes, that is, knowledge.

Define classes Context, Word, Phrase, Sentence. Define different attributes for different classes: part-of-speech attribute (hasPartOfSpeech) for Word class, word attribute( hasWord) for phrase and sentence class, phrase attribute(hasPhrase) the context attribute (forContext ) for Sentence class.

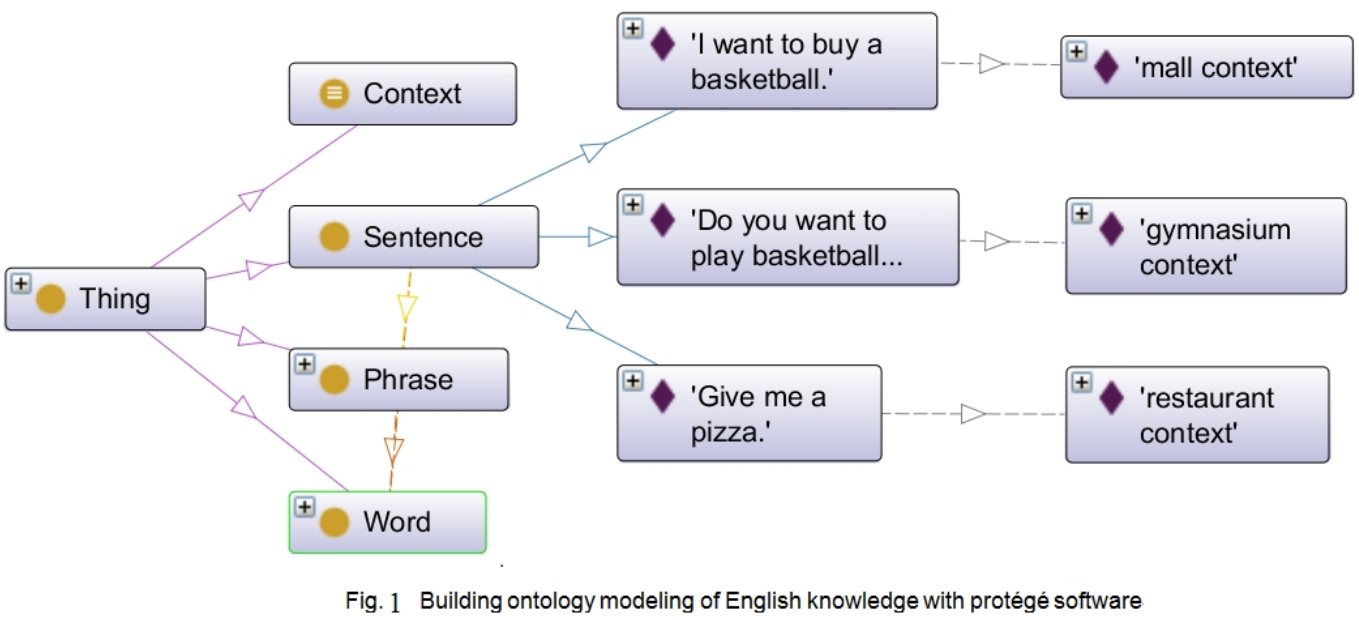

The fragment of implementation code is shown as follow.

<owl:Class rdf:about="\#Sentence">

<rdfs:subClassOf> 


\author{
<owl:Restriction> \\ <owl:onProperty rdf:resource="\#forContext"/> \\ <owl:hasValue rdf:resource="\#Context"/> \\ $</$ owl:Restriction> \\ $</$ rdfs:subClassOf $>$ \\ $<$ rdfs:subClassOf $>$ \\ <owl:Restriction> \\ <owl:onProperty rdf:resource="\#hasPhrase"/> \\ <owl:allValuesFrom rdf:resource="\#Phrase"/> \\ $</ o w l: R e s t r i c t i o n>$ \\ $</$ rdfs:subClassOf $>$ \\ $<$ rdfs:subClassOf $>$ \\ <owl:Restriction> \\ <owl:onProperty rdf:resource="\#hasWord"/> \\ <owl:allValuesFrom rdf:resource="\#Word"/> \\ $</$ owl:Restriction> \\ $</$ rdfs:subClassOf $>$ \\ $</$ owl:Class $>$
}

The following program fragment defines a sentence, "Do you want to play basketball?" Which is suitable for pushing in a stadium context, including the phrase "play basketball" etc.

$<$ Sentence rdf:ID="sentence002">

<rdfs:comment> nǐ xiăng dă lán qiú ma ? </rdfs:comment>

<rdfs:label>Do you want to play basketball?</rdfs:label>

$<$ hasPhrase rdf:resource="\#play_basketball"/>

$<$ hasWord rdf:resource="\#basketball"/>

$<$ hasWord rdf:resource="\#play"/>

$<$ forContext rdf:resource="\#gymnasium"/>

$</$ Sentence $>$

\title{
System Design
}

System Design Objectives. After review on the research about informal learning context and English knowledge in mobile Internet, and study the correlation algorithm, we designs a context aware English knowledge push APP based on LBS and put into use.

Once a user entered the system-known area, the server will automatically inferred out the current context the user is in, then query for the English knowledge utilizing the user's interests and history data( feedback, etc.), such as word, phrase and sentence, finally push to the mobile-end immediately.

System Architecture. The system is designed with multi-layers architecture, which consists of data layer, business layer, service layer and data layer, from top to bottom. The system architecture is shown in Fig.2. The application layer obtains the current location of the user through the mobile phone and submits it to the service layer. It receives the context-related English knowledge pushed from business layer in real time and presents it to the user to interact. The business layer invokes the location service and POI query service of the service layer to analyze the context according to the current position of the user. After detected a new context, the service layer will invokes the message push service to push the recommend English knowledge to user. The service layer contains location 
query service, POI query service and message push service. Location query service and POI query are based on third-party LBS. Message push service are based on third-party message push platform. The data layer includes the context knowledge base, as well as context profiles and user profiles. The context profile and context-related knowledge base are used to provide context-sensitive English knowledge for user. The user profile is intended to provide a personalized learning service for user.

The context information of the user's geographic location is used to provide the personalized context corpus. The end-user's geographic locations are perceived through the positioning service provided by Android mobile phone (support GPS, WLAN and mobile network, etc.). The Android map SDK provided by Gaode Open Platform is used to carry out geocoding and point-of-interest (POI) search. Using the cloud push (pigeon) SDK provided by Tencent open platform, it's easy to achieve fast, accurate message push.

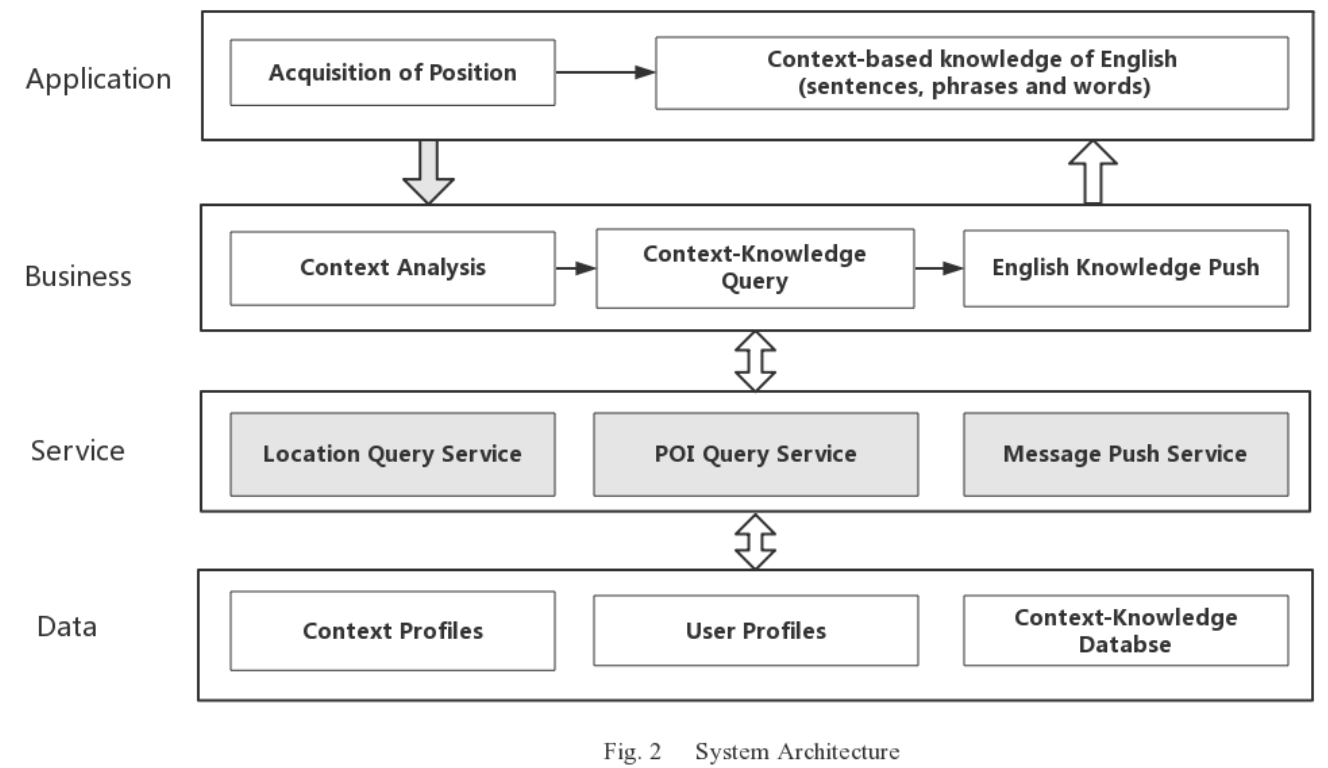

\section{Critical Technology}

Location-aware. Mobile phone systems and satellite navigation systems provide location-aware support at the regional and global scales, and the corresponding terminal devices are mobile phones and GPS receivers.

The mobile phones can realize location-aware by GPS and Wi-Fi, which is relatively low accuracy, but is real-time and dynamic continuously.

There have been Gaode map and Baidu map, which is the excellent location service solutions provider in China which provides the free android development interface (API) to makes it become possible to realize location-aware.

Build Context Profile. The mainstream map software provides deep POI query function. The results of POI query no longer just a pile of place names, but a virtual reality. POI list contains at least three aspects of information: name, type, distance.

One POI list will be returned by Gaode map when the Android SDK is called. Each item of POI list can be divided into 6 parts: index, locations name, the first-level category, the second-level category, the third-level category, distance and district. The fragment of POI list is showed as follow.

1. Baishi Express: life service; express service; express service, 6, Panyu district,

2. Hachashijia: catering services; drink shop; drink shop; 34, Panyu district, 
3.Wangjia: catering services; western restaurant; western Restaurant, 88, Panyu district,

The contexts are classed based on English learning needs. For example, although western restaurant and drink shop are belonged to catering services in POI lists, we have built western restaurant and drink shop context classification. We have designed context profile to record the relationship between the context classification name and POI.

The context profile is shown as follow.

\{context classification name: the first-level category of POI, the second-level category of POI \}

Context-aware Based on POI Query. We use search API of Android map SDK, which is provided by the open development platform of Gaode map. Gaode map provides a number of 10 million of POI. POI can represent a building, a shop, a spot and so on in the map. By the query for POI, we can find the restaurants, attractions, and toilet and so on. Search SDK provides a variety of interfaces to retrieve POI, including the keyword search POI, retrieval of the peripheral POI, retrieval of polygons within the POI and so on.

In order to find the POI where the user is in, we need to retrieve the peripheral POI to further determine the current context. POI search around the search for, such as the Guangzhou University, near the west restaurant and other scenes, the results also include the POI category, such as food and beverage category.

The critical algorithm is shown as follow.

1) Gets the latitude and longitude of the user's location

2) Select the POI category you want to query (if you do not select POI category, system return three categories of POI: "restaurant service", "commercial housing", and "life services")

3) Select circle query range (unit: $m$ )

4) Call the POI search API to retrieve the surrounding POI and wait for the search results (asynchronous)

5) After the search is completed, search the context profile in the callback function according to the name and category of POI

6) Return the current context where the user is in to the system

The critical code (in java) is shown as follow.

query = new PoiSearch.Query("", "", cityCode);

poiSearch = new PoiSearch(this, query);

poiSearch.setBound(newSearchBound(newLatLonPoint(locationMarker.getPosition().latitu

de, locationMarker.getPosition().longitude), 10));

poiSearch.setOnPoiSearchListener(this);

poiSearch.searchPOIAsyn();

// callback function

public void onPoiSearched(PoiResult result, int rCode) \{

dissmissProgressDialog();

if ( $\mathrm{rCode}==1000)\{$

if (result != null \&\& result.getQuery() != null) \{

if (result.getQuery().equals(query)) \{

List $<$ PoiItem $>$ poiltems $=$ result.getPois () ;

if (poiItems $!=$ null $\& \&$ poiltems.size ()$>0$ ) \{

Poiltem poi $=$ poiItems[0];

// TODO Query context profile according to the POI name

// TODO Report the context to system 


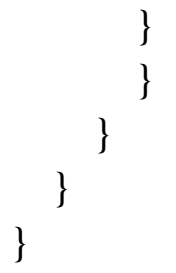

Message Push. XG Push of Tencent is a free professional mobile App push platform which supports message push; with it the messages can reach mobile users in one second. ${ }^{[10]}$ We use the SDKs including the client-side and the server-side provided by XG Push to push the suggested English knowledge to the user instantly. The client-side SDK is used to receive messages and the server-side SDK is used to push messages.

The critical algorithm of APP program:

1) Set AccessId and AccessKey in Androidmanifest.xml

2) Set receiver in Androidmanifest.xml, specify the class to receive message (derived from XGPushBaseReceive, and override onTextMessage method)

3) Call regist interface registerPush to complete start and regist operation。 If success, return device token

4) Process the receive message in the recall function "onTextMessage"

The critical code(in java) is shown as follow.

public void onTextMessage(Context context, XGPushTextMessage message)

\{

String title $=$ message. getTitle () ;

String content $=$ message. getContent () ;

String fields = message. getCustomContent () ;

// TODO According to the obtained field table, we extract English knowledge and present it to the end-user.

\}

Server-side interface using Rest API, data in json format. The interface URL of pushing messages to specific devices : http://<host $>/ v 2 /$ push/single_device?params. Params specific content (background sent by POST push platform) is as follows:

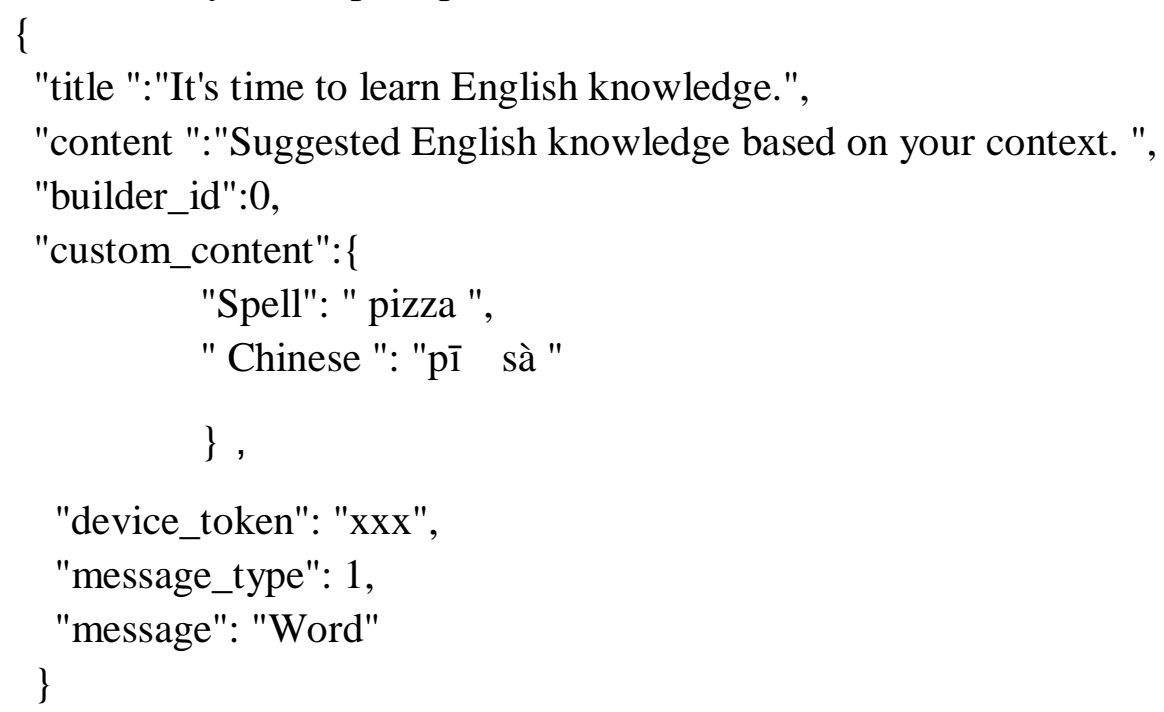

Knowledge Query Based on SPARQL. The Simple Protocol and RDF Query Language (SPARQL) is a SQL-like language for querying RDF data. ${ }^{[11]}$ 
SPARQL can be used to express queries across diverse data sources, whether the data is stored natively as RDF or viewed as RDF via middleware. SPARQL contains capabilities for querying required and optional graph patterns along with their conjunctions and disjunctions. SPARQL also supports extensible value testing and constraining queries by source RDF graph. The results of SPARQL queries can be results sets or RDF graphs. ${ }^{[12]}$

Queries the English sentences that fit the given context:

SELECT ?sentence ?chinese ?phrase

WHERE \{

?sentence rdf:type owl:Sentence.

?sentence rdfs:comment ?chinese.

?sentence owl:forContext ?context.

FILTER(regex(str(?context), "mall")) \}

\section{Conclusions}

With the popularity of mobile devices, the Mobile-Learning software which is adopted the context-aware technology will become the most popular tool for people to study in the future. A context-aware English knowledge push APP based on LBS is designed, based on the review of the research and algorithm of OWL,OPI,LBS,SPARQL and message push , and it was successfully applied in test. The test proves that learning in a specific context can enhance the user's understanding of the English knowledge, thus deeply comprehending the English knowledge, and improve the ability of using English. However, the data of context database and context-knowledge database is not enough, need to increase the amount of data, in addition, the software algorithm also needs to be further optimized to improve its ease of use.

\section{Acknowledgements}

This paper was financially supported by 2014 the humanities and social sciences research project of Ministry of Education - "Research on micro learning environment of personal knowledge service based on the location " (Item number: 14YJC880031), 2015 innovative training project for College Students of Guangzhou University -" Research on context-Aware English Learning System based on LBS"(Item number: 201511078020)

\section{References}

[1] Juan Li, Biao Liu, Xinpeng Wang and Xianmei Lu : Modern Educational Technology Vol.19.87-89(2009)

[2] Ming Yi et al.: Journal of Library Science in China Vol 37.4-12(2011)

[3] Feng Xiao, Yue Hou, Liuzhao Wang and Bao Jia: Geomatics Spatial Information Technology Vol.38.125-127(2015)

[4] Yalong Liu: E-education Research Vol .7.113-116(2015), p. 120

[5] Hongjian Liao, Zhe Qu: Modern Educational Technology Vol .24:92-99(2014)

[6] Information on http://tc.people.com.cn/n1/2016/0803/c183008-28606650.html

[7] Deborah L McGuinness, Frank van Harmelen: OWL web ontology language overview(2005)

[8] Michael K Smith, Chris Welty, Deborah L McGuinness: OWL web ontology language guide(2004)

[9] Thompson H S: XML schema part 1: Structures (2005) 
[10] Yu Liu, Yu Xiao, Song Gao, Chaogui Kang and Yaoli Wang: Geography and Geo-Information Science Vol.27:8-13(2011)

[11] Information on

http://www.obitko.com/tutorials/ontologies-semantic-web/rdf-query-language-sparql.html [12] Information on https://www.w3.org/TR/rdf-sparql-query/ 